\title{
Li-Fi a Green Energy Initiative
}

\author{
Balaram Ghosal \\ BPPIMT, Kolkata, India
}

\author{
Asim Kumar Panda \\ BPPIMT, Kolkata, India
}

\begin{abstract}
The world as on date revolutionized by the use of internet, the number of devices accessing the internet is growing by second. Most of the devices use wireless communication for this; now this has unfortunately led to an increase in network complexity, shortage of wireless radio bandwidth and an increased risk of interference of radio frequencies. Moreover speed and security of the internet are major issues. Present paper reflects the Future of Wireless Communication (LI-FI) which may affect all lives. LiFi (Light-Fidelity) is designed to use LED light bulbs as the mode of communication which is used equally to light up the places as energy saver lighting. Speed, security, reliability is also maintained. It is a technology more powerful to the same idea as on infrared remote controls. Experimentally LiFi gives a speed of 500MBPS. The visible light is the future of data communication.
\end{abstract}

\section{Keywords}

Wireless-Fidelity (Wi-Fi), Light-Fidelity (Li-Fi), Light Emitting Diode (LED), Photo Diode.

\section{INTRODUCTION}

The exponential increase in mobile data traffic during the past two decades has led to the massive deployment of wireless systems. As per the Cisco Survey of the usability of the existing spectrum, we are presently using about $80 \%$ of the existing capabilities of the data utilization. Presently we are utilizing the Wi-Fi services within the campus and around the 10-100 meter distances to connect P.C., Laptops, tab and smart phones etc. It is estimated that by 2017, more than 11 Exabyte of data traffic will have to be transferred through wireless networks every month. Therefore the expanding demand for wireless data has led to clogging of the radio spectrum. Li-Fi is the new prototype suggested by Dr.Harald Haas of University of Edinburgh, UK, that overcomes the shortcomings of Wi-Fi. Li-Fi is a term of one used to describe visible light communication technology applied to high speed wireless communication. It acquired this name due to the similarity to $\mathrm{Wi}-\mathrm{Fi}$, only using light instead of radio frequency.

\section{BASIC PRINCIPLE}

The idea behind LiFi first presented by Professor Harald Haas from University of Edinburgh, UK, in his TED Global talk on Visual Light Communication. He explained, "Very simple, if the LED is on, you transmit a digital 1; if it's off you transmit a 0 . The LEDs can be switched on and off very quickly, which gives nice opportunities for transmitting data." [1] The off-on process of LED is completely undetectable on human eye. LED is the choice for light source as it consumes very less power when compared to fluorescent lamp or a light bulb. It consumes about one-tenth the power when compared to conventional methods of lighting. Also, the lifetime of a typical LED bulb is very high. Fast switching off-on is also supported by LED lamp. Basically LED is used as the sender of data, encoded in the light by varying the rate at which the LEDs flicker on and off to give different strings of $1 \mathrm{~s}$ and $0 \mathrm{~s}$. The receiver section consists of a Photodiode, e.g. silicon photo detector or an Infrared germanium cylindrical detector. The Photodiode demodulates the incoming received signal based on the sequence of $1 \mathrm{~s}$ and $0 \mathrm{~s}$.

\section{IMPLIMENTATION}

$\mathrm{Li}-\mathrm{Fi}$ communication is structured according to communication Protocols set forth by the IEEE 802 workgroup. The proposed plan is to establish a wireless network using visible light. The visible light is termed as an electromagnetic radiation in the range of wavelengths from about 390 to $700 \mathrm{~nm}$ corresponds to a frequency band of 430 $790 \mathrm{THz}$. The Internet data is first converted to electrical signal using some signal converter, and then a microcontroller is used according to the converted signal to modulate the LED lamp off and on. As a result the LED is produce flicker which is hidden to human visibility. A set of Photo Diode convert the flickering light to some digital data signal. This signal can be further filtered and amplified to get the desired Internet data. Thus a Li-Fi network is established.

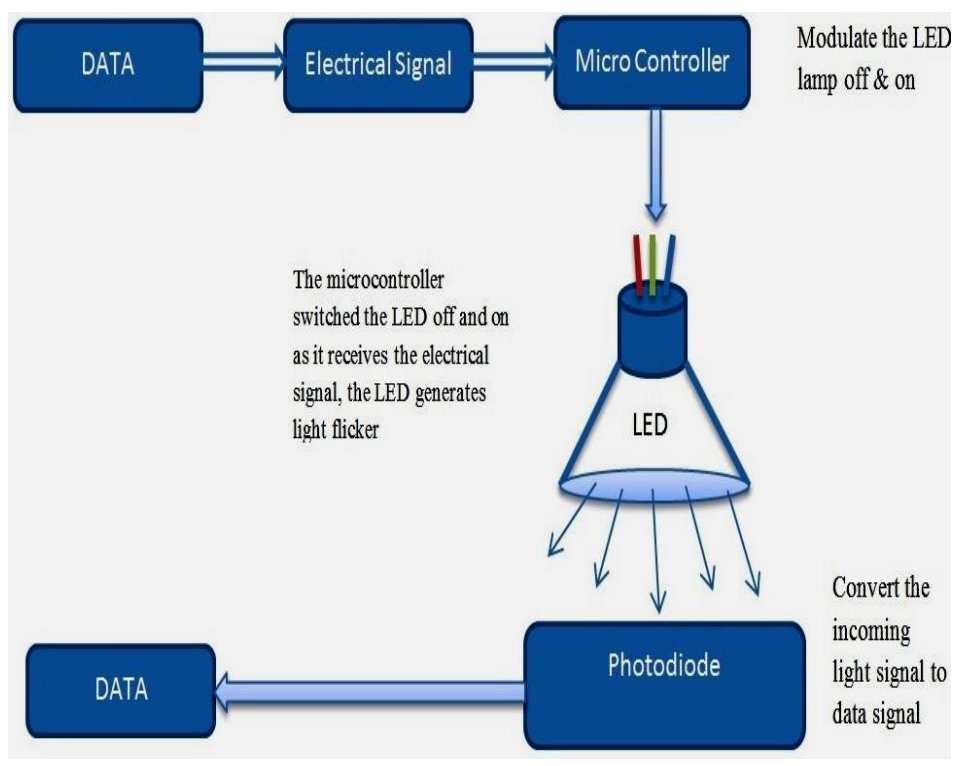

Fig.1: System Block Diagram 
1000 Base

T Ethernet

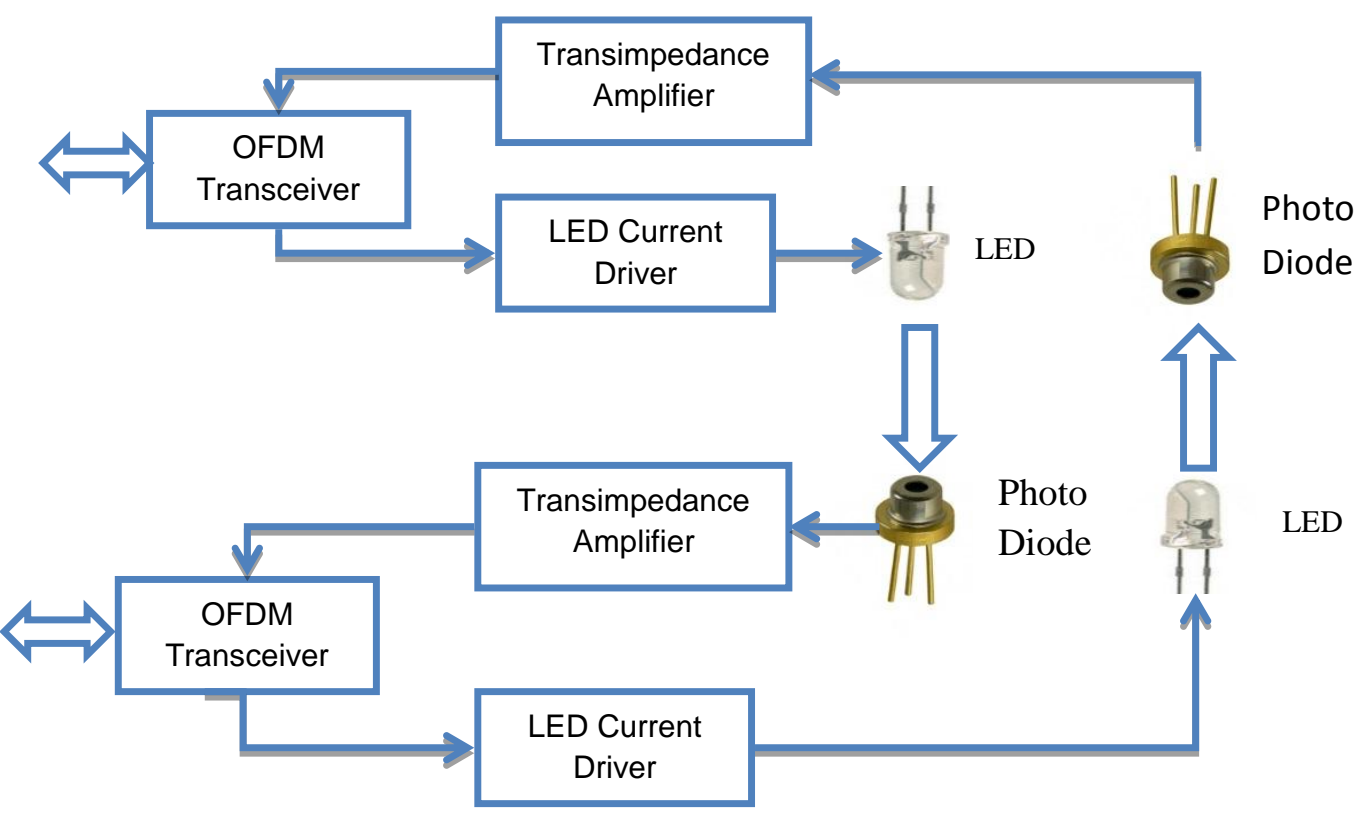

Fig. 2: Full Duplex Li-Fi Communication

Uplink Mechanism: As a future replacement of Wi-Fi Technology Li-Fi must provide a full duplex communication. Till now most of the research works were done on the downlink transmission of data from the optical Access Points. There must be an uplink procedure from devices to the Access Points. For uplink purpose different colour LED may be used. Photo diode embedded on Li-Fi access points will catch the light pulse form those LED. As a modulation technique Orthogonal Frequency Division Multiplexing (OFDM) may be used, where a high data rate serial data stream is split up into a set of low rate sub-streams. The total channel bandwidth is divided into a number of orthogonal frequency sub channels. Each low rate sub-stream is modulated on a separate sub channel [2] [3].The orthogonality is achieved by selecting a special equidistant set of discrete carrier frequencies. Infrared transmission is another alternative for uplink transmission.

\section{PROPOSED DEVICES}

This paper proposed two devices for better $\mathrm{Li}-\mathrm{Fi}$ communication; 1. Li-Fi Access Point and 2. Li-Fi Dongle. For smart mobile devices the flash LED may be taken as the data sender and the camera may be operate as a data receiver. The Infrared port as well can be the data sender, but with considerably low speed compared to the LED light.

\subsection{Li-Fi Access Point}

The Li-Fi Access Point as designed with an array of white LED embedded with silicon or germanium cylindrical photo diode, where the array of LED and multiple Photo Diode worked as a MIMO(multiple input multiple output). The array of LED light is used to produce many different data stream from a single access point simultaneously. Parallel access is possible through this access point.

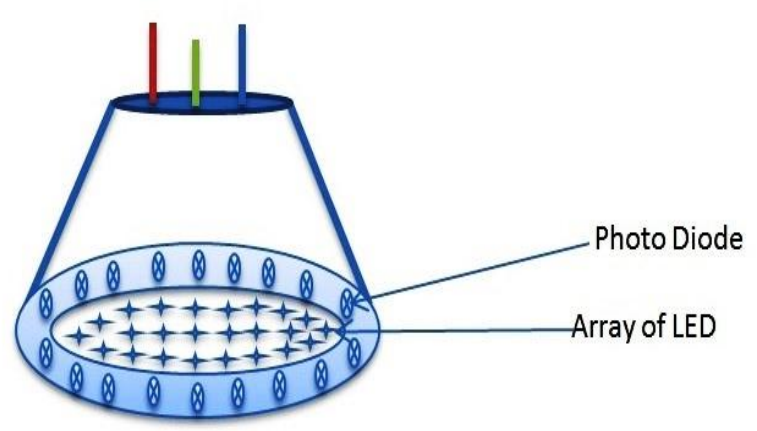

Fig. 3: Li-Fi Access Point

\subsection{Li-Fi Dongle}

As designed similarly with an array of Led and a set of photo diode embedded in an USB dongle with data modulation and demodulation capability. On Similar manner this dongle can be used with a simple USB port. Here same color LED light should not be used as in the Li-Fi access point; rather some different color LED (say red, green and blue) may be used, embedded with typical prism to produce the uplink light signal. Here using three colors LED it is possible to encapsulate the light signal to send encoded data. The Light from this dongle after moving through the prism it is possible to form a visually white light but logically different from the light coming from the Li-Fi access point. 


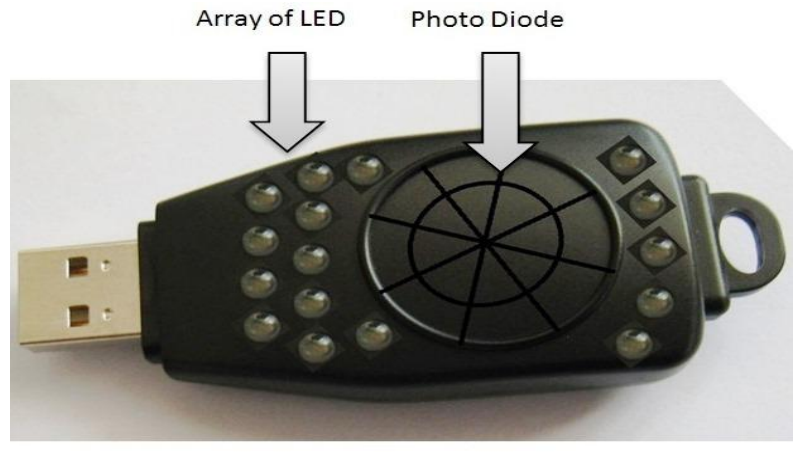

Fig. 4: Proposed Li-Fi Dongle

\section{COMPARISION WITH OTHER WIRELESS MEDIA}

$\mathrm{Li}-\mathrm{Fi}$ is acquired this name due to the similarity to $\mathrm{Wi}-\mathrm{Fi}$, only using light instead of radio frequency. So this technology can easily be used in the places where Bluetooth, infrared, Wi-Fi cannot be used. Wi-Fi is very good for general wireless coverage area within building; but many simultaneous users in one place lead to lesser speed. Li-Fi is ideal for high density wireless data coverage in confined area. Moreover no one has to pay to use the radio bandwidth, as light is open to use anywhere. In Li-Fi many different colour light may be used to communicate secured data, the advantage is you are doing Internet as well you enlighten and decorate your place. The comparative technicalities of the existing and proposed wireless media are enlisted below considering Wi-Fi, Bluetooth, Infrared and proposed Li-Fi communication.

\begin{tabular}{|l|l|l|l|l|}
\hline Parameter & Li-Fi & Wi-Fi & Bluetooth & IrDA \\
\hline Speed & $>1 \mathrm{Gbps}$ & $\begin{array}{l}150 \\
\text { Mbps }\end{array}$ & $3 \mathrm{Mbps}$ & $4 \mathrm{Mbps}$ \\
\hline $\begin{array}{l}\text { Data } \\
\text { Density }\end{array}$ & High & Low & Low & Low \\
\hline Security & High & Medium & Low & High \\
\hline $\begin{array}{l}\text { Power } \\
\text { usage }\end{array}$ & Low & Medium & Low & low \\
\hline $\begin{array}{l}\text { Ecological } \\
\text { impact }\end{array}$ & Low & High & Low & Low \\
\hline $\begin{array}{l}\text { Cost of } \\
\text { material }\end{array}$ & High & Medium & Low & Medium \\
\hline $\begin{array}{l}\text { Market } \\
\text { maturity }\end{array}$ & $\begin{array}{l}\text { Newest } \\
(2011)\end{array}$ & $\begin{array}{l}\text { Old } \\
(1990)\end{array}$ & $\begin{array}{l}\text { New } \\
(1998)\end{array}$ & Old(1917) \\
\hline
\end{tabular}

\section{Table1: Comparison with Li-Fi \& others}

\section{USAGE \& LIMITATION OF Li-Fi}

Li-Fi is a high intensity light source of revolutionary design. This technology does not deal with radio waves, so it can be easily be used in the place where Bluetooth, infrared, Wi-Fi cannot be used. The major areas are:

RF Spectrum Relief: Excess capacity demands of cellular networks can be off-loaded to Li-Fi networks where available. This is especially effective on the downlink where bottlenecks tend to occur.
Hazardous Environments: Li-Fi provides a safe alternative to electromagnetic interference from radio frequency communications in environments such as mines, petrochemical plants, nuclear power plant etc.

Hospital \& Healthcare: Li-Fi emits no electromagnetic interference and so does not interfere with medical instruments, nor is it interfered with by MRI scanners.

Smooth Trafficking and Transportation: Using LED light on traffic it is easy to collect traffic data and accordingly may be smoothen, Car embedded with LED lamp can communicate with each other, as a result chances of collision is minimized.

Underwater Communications: Radio wave is strongly absorbed in water, so using this is impractical. Acoustic waves have extremely low bandwidth and disturb marine life. $\mathrm{Li}-\mathrm{Fi}$ provides a solution for short-range communications under water.

Location Based Services: Highly accurate location-specific information services such as advertising and navigation that enables the recipient to receive appropriate, pertinent information in a timely manner and location.

However this technology also has some limitation as enlisted below:

1. Only works if there is direct line of sight (LOS) between the transmitter and receiver.

2. Data transmission can be easily obstructed by opaque obstacles.

3. The use of very high frequencies $(400-800 \mathrm{THz})$ limits it to very short distances and point to point communication only.

4. Interference from external light sources like sun light, normal bulbs in the path of transmission will cause interruption in the communication.

5. A high speed moving object and to provide data in a remote area where there are trees and walls is unreliable to use this technology.

\section{CONCLUSIONS}

With the development of this technology every bulb (LED) can be used something like a hotspot to transmit wireless data and the globe will proceed toward the cleaner, greener, safer and brighter future. $\mathrm{Li}-\mathrm{Fi}$ is already tested in different lab as a downlink media at a very high speed, if used LED of different colour as the uplink media then the problem due to signal interference also be removed. Large scale areas that are saturated with radio signals or that doesn't permit them for security reasons could use $\mathrm{Li}-\mathrm{Fi}$ as an alternate high-speed wireless network solution. The traffic system all over the globe may be revolutionized if all the light used in traffic signal and in cars is converted to LED access point and receiver, which is the biggest aspect of this technology. Data hand off over different access points and data upload on a very high speed is the biggest challenge to this technology.

\section{REFERENCES}

[1] Haas,Herald(July 2011).”Wireless data from every light bulb".TED Global. Edinburgh .Scotland

[2] Tsonev, D.; Sinanovic, S.; Haas, Harald (15 September 2013). "Complete Modeling of Nonlinear Distortion in OFDM-Based Optical Wireless Communication". IEEE Journal of Lightwave Technology 31 (18): 3064 3076.doi:10.1109/JLT.2013.2278675 
[3] Y. Tanaka, T. Komin, S. Haruyamaand, and M. Nakagawa, "Indoor Visible Communication Utilizing Plural White LEDs as Lighting," in Proceedings of the 12th IEEE International Symposium on Personal, Indoor and Mobile Radio Communications (PIMRC 2001), San Diego,CA, September 2001, pp. F81-F85.

[4] http://en.wikipedia.org/wiki/Li-Fi

[5] http://purelifi.com/what_is_li-fi//what_is_li-fi

[6] “ Visible-light communication: Tripping the light fantastic: A fast and cheap optical version of Wi-Fi is coming”, Economist, dated 28Jan 2012

[7] The-gadgeteer.com/2011/08/29/li-fi-internet-atthespeed-of light.

[8] Will Li-Fi be the new Wi-Fi?. New Scientist, by Jamie Condliffe, dated 28 July 2011
[9] Jia-yuan WANG, Nian-yu ZOU, Dong WANG, Kentaro IRIE, Zensei IHA, Yoshinori NAMIHIRA," Experimental study on visible light communication based on LED”, The Journal of China Universities of Posts and Telecommunication,vol. 19, Supplement 2, October 2012.

[10] Dominic O’Brien, Hoa Le Minha, LubinZeng, Grahame Faulkner and HsiHsir Chou, Kyungwoo Lee, Daekwang Jung, YunJe Oh, Eun Tae Won,"Visible Light Communication: Recent Progress and Challenges", Wireless World Research Forum.

[11] http://visiblelightcomm.com/an-ieee-standard-forvisible-light-communications/

[12] http://www.lificonsortium.org/. 